\title{
Real-time Analysis of City Scale Transportation Networks in New Orleans Metropolitan Area using an Agent Based Model Approach
}

\author{
Millard McElwee ${ }^{1, *}$, Bingyu Zhao $^{2}$, and Kenichi Soga ${ }^{1}$ \\ ${ }^{1}$ Department of Civil Engineering, University of California Berkeley, CA 94720 \\ ${ }^{2}$ Department of Civil Engineering, University of Cambridge, United Kingdom
}

\begin{abstract}
The primary focus of this research is to develop and implement an agent-based model (ABM) to analyze the New Orleans Metropolitan transportation network near real-time. ABMs have grown in popularity because of their ability to analyze multifaceted community scale resilience with hundreds of thousands of links and millions of agents. Road closures and reduction in capacities are examples of influences on the weights or removal of edges which can affect the travel time, speed, and route of agents in the transportation model. Recent advances in high-performance computing (HPC) have made modeling networks on the city scale much less computationally intensive. We introduce an open-source ABM which utilizes parallel distributed computing to enable faster convergence to large scale problems. We simulate 50,000 agents on the entire southeastern Louisiana road network and part of Mississippi as well. This demonstrates the capability to simulate both city and regional scale transportation networks near real time.
\end{abstract}

\section{Introduction}

Agent Based Models (ABM) have received widespread popularity in recent years across a variety of academic disciplines (transportation, building evacuation, biology, economics, policy, game theory, etc.). There seems to be a growing consensus that $\mathrm{ABM}$ represents the optimal choice for modeling complex system dynamics at the city scale [1]. According to the United Nations, $55 \%$ of the world's population lives in urban areas, and this is expected to increase to $68 \%$ by 2050 [2]. This rapid shift towards a more congested infrastructure requires a complex fine resolution framework that can handle a bottom-up modeling approach capable of observing macro-level interactions (i.e., flooding, earthquakes, hurricanes).

Depending on the field, the definition of what an $\mathrm{ABM}$ is can drastically change. Because of this possible ambiguity, we use the definition of Jennings: "An agent is a computer system, situated in some environment, that is capable of flexible autonomous action in order to meet its design objectives" [3]. This paper presents an open source system level architecture in Python capable of simulating communities at the city and region scale. A case study of Metropolitan New Orleans was selected to eventually observe the influence of natural hazards on one of the most susceptible places to destruction because of its geographic location. These fine scale resolution micro-simulations have only very recently (within the last decade) been possible due to advancements in highperformance computing (HPC) [4].

\section{Literature review}

The goal of this brief literature review is to understand current techniques and trends used for large scale ABM geospatial simulations of transportation networks. We begin with the evolution of $\mathrm{ABM}$, discuss trends in $\mathrm{ABM}$ for traffic simulation, and conclude with challenges and future disruptions.

\subsection{Emergence and evolution $A B M$ for road network}

The emergence of ABMs can be traced back as early as 1940 based on the work of well-known mathematician and computer scientist Von Neuman. He is credited with creating a device coined the "cellular automata", which simulated grid interactions with immediate neighbors of cells [5]. In the 1970's John Conway took it a step further and created the "Game of Life" based on Von Neuman's work. In this game, a cell has two states (alive or dead) which depend on the state of the neighbors in the previous time step [6]. Computer simulations were revolutionized yet again with Craig Reynolds' Boids agents that had the ability to bring intelligence and behavior to the model [7]. With Reynold's contribution, macro level emergent behaviors were simulated for the first time.

ABMs became increasingly popular and adopted in a variety of academic fields in the 1990s when several companies started making commercially available

\footnotetext{
* Corresponding author: mcelwee@berkeley.edu
} 
software for ABM simulations [8]. The advantage of our framework over many of the commercially available ABM tools is the flexibility, performance, and trafficspecific nature of our model. Traditionally, ABM traffic simulations have used a deterministic top-down approach which is not capable of capturing the complex systems in a way that bottom up stochastic methods can [1]. Packages such as OSMnx have made the extraction of road networks (length, lane count, and road type) fast with the use of standard functions and protocols [9]. The use of limited, old and context specific data such as traffic counts, volume-delay curves, and Annual Average Daily Flows (AADFs) are not always capable of capturing accurate traffic behavior over long periods at the same accuracy that Google Directions Application Program Interface (API) can. This API is able to harvest GPS informed journey travel times from roads all across the world.

\subsection{City scale transportation networks challenges and future disruptions}

GIS and associated functions have the capacity to perform spatial analysis (queries, shortest path, observe patterns, recognize trends, etc.) and real time visualization of transportation networks [10]. Big data has resulted in the tracking and storage of more Global Positioning System (GPS) data than we know what do with [11]. This data ranges from road sensors to obtain traffic counts or data logs obtained from cell phone tracking movement over longer periods of time. ABMs can import multimodal transportation (rail, metro, water, bus, etc.) anywhere with publicly available data such as General Transit Feed Specification (GTFS). Incorporating different modal weights in combination with discounts such as socio-economic status, time of day, traveler's purpose, and even weather can affect the travel decision of the traveler [12].

One major advantage of ABM transportation networks is the ability to incorporate outside complex travel demand behavior models [13]. Understanding how the interplay of number of vehicles, available road capacity, and routes impact level of congestion is import for accurately simulating travel in these urban areas. ABM with GIS applications are growing in popularity in AI (inverse reinforcement learning) and game theory. Recent studies have shown it is possible to observe the influence of peer pressure on reducing congestion and emissions [14].

\section{Methodology}

There are two main schemes used to create and validate the ABM of our transportation network. The first scheme requires harvesting road network data from OSM, obtaining/assuming travel demand data, and producing ABM simulations. Figure 1 highlights the main steps of the first scheme used in the New Orleans transportation network. The traffic model used to create the network, demand, and agent-based simulation for New Orleans was derived from the open source GitHub repository: https://github.com/cb-cities/sf_abm which is an ABM for the city of San Francisco. There are three main folders which correspond to the main steps in Figure 1.

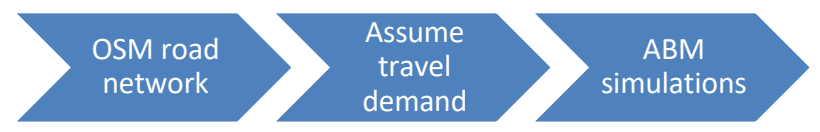

Fig. 1. ABM framework used for New Orleans transportation network.

The first step is querying road network data from OSM using a bounding box for the desired New Orleans metropolitan area. The script then cleans the OSM data by removing "curve" nodes that do not define edge intersections, converts OSM data to GeoJSON for easy visualization, and prepares a python-igraph or sparse matrix for shortest path calculations. The final graph has a total of 483,794 edges and 205,332 nodes. The second step requires constructing an hourly Origin Destination (OD) matrix for the desired location. The San Francisco ABM uses Uber/Lyft pick-ups and drop-offs in the city by Traffic Analysis Zone (TAZ) to generate OD pairs. Due to time constraints, 50,000 OD pairs, or travel demand were randomly generated in this study. This is not necessarily representative of hourly demand on a weekday morning, but it is acceptable size for testing city-scale modeling. However, future study will consider OD pairs of the area. The final step is running the shortest path calculation for each OD pair. Here Dijkstra's Algorithm is used for implementation of the shortest path between nodes in a graph.

The second scheme requires harvesting google directions API, processing this data, and visualizing city level travel time. The research focus for this project was on the first scheme, and the second scheme is intended to calibrate or validate the ABM simulations.

\section{Case Study}

The New Orleans metropolitan area is a desirable location for a case study for several reasons. One is its size with a total of eight parishes and population of $1,337,726$ based on the 2000 census. Secondly, New Orleans is one of the most resilient cities in the world and has sustained an estimated $\$ 300$ billion in repairs to infrastructure, more than 60,000 homes destroyed, and losses in excess of 1,300 lives from Hurricane Katrina alone in 2005 [15]. In a recent analysis of 27,000 urban street networks in every US city, town, urbanized area, and Zillow neighborhood, New Orleans was identified as the densest city with 49.4 intersections per $\mathrm{km}^{2}$. It has high proportions of 4-way intersections, and older neighborhoods like the French Quarter have predominantly orthogonal grids [16]. Finally, New Orleans' access and importance of the Mississippi River make it an ideal location to explore the interactions of various networks. 
The road networks shown in Figures 2 and 3 were created with OSM and confirm many of the claims about New Orleans' unique transportation network. The New Orleans road network in Figure 2 consists of Orleans' parish road network and has a very dense network that spreads outward from the center. Also, the 1 square mile block of downtown New Orleans has many 4 ways and an overall orthogonal structure.

Fig. 2. New Orleans road network.

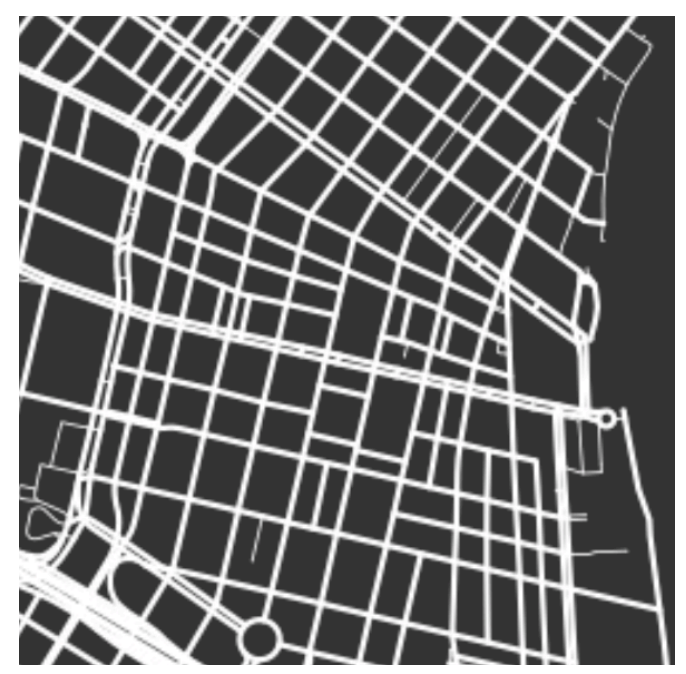

Fig. 3. Square mile of downtown New Orleans.

The final step is simulating agent movement based on shortest path calculations for the randomly generated 50,000 OD pairs and visualizing these movements via graph. The ABM output file is a GeoJSON with section properties that include: link identification number, section speed, section volume, start and end coordinates. The final network consists of 483,794 edges and 205,332 nodes. Visualization is done in QGIS 3, a free and open source geographic information system. Figure 4 shows 50,000 agents on the road network with blue links corresponding to section volumes near zero and red links corresponding to section volumes with more than 50 agents. This terrain map overlaid with the transportation network shows the capabilities of the ABM to model city and regional scale transportation networks for the entire southeastern Louisiana road network.

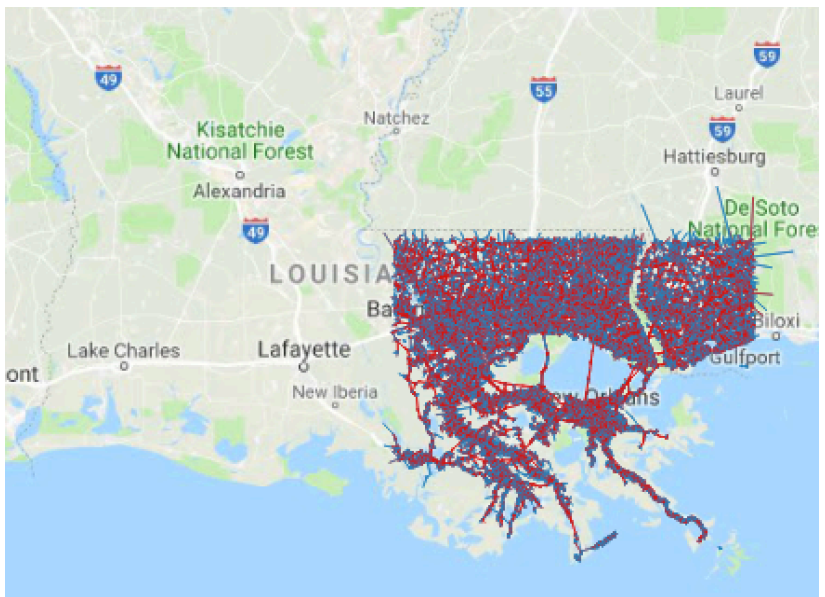

Fig. 4. 50,000 agents simulated on southeastern Louisiana road network.

\section{Next steps}

After simulating transportation of the studied area using randomly generated ODs, the next step is to create an OD matrix that accurately depicts the travel demand for this region. One way may be to use a hybrid approach (meso-simulation model for local traffic and a microsimulation model for highway traffic) to construct OD matrixes for New Orleans [17].

Macro-scale natural hazard events such as inundations impact the weights of edges on a graph network. Road closures and reduction in capacities are examples of influences on the weights or removal of edges which can affect the travel time, speed, and route of agents in the transportation model. In the Bay Area, high resolution numerical simulations have shown promise for studying the impact of sea level rise using hydrodynamics models [18]. The future study will use a hydrodynamic model to study the impact of extreme rain events such as 100-year flood as well as storms such as high category hurricanes on the Greater New Orleans metropolitan area.

\section{Conclusions}

The overall aim of this paper is to present an $\mathrm{ABM}$ framework with the capacity to model a city scale transportation network for the Greater New Orleans metropolitan area. The final result was simulated transportation dynamics for the entire southeastern Louisiana. This includes the Greater New Orleans metropolitan area in addition to stretching from Baton Rouge, LA to Gulfport, Mississippi. The python code is currently converted to $\mathrm{C}++$ to speed up convergence to solution of even larger complex interdependent networks (i.e., water pipe network). Modeling these complex systems in a bottom up stochastic method allows 
transportation planners to better understand agents' decision making under varying degrees of uncertainty.

\section{References}

1. Casey, G., Soga, K., Silva, E., Guthrie, P., and Kumar, K. (2017). A Scalable Agent Based Multimodal Modeling Framework Using Real-Time BigData Sources for Cities.

2. United Nations, and Department of Economic and Social Affairs. (2014). World urbanization prospects, the 2014 revision: highlights.

3. Jennings, N.R., Sycara, K., and Wooldridge, M. (1998). A Roadmap of Agent Research and Development. Autonomous Agents and Multi-Agent Systems, 1, 7-38.

4. Soga, K., Casey, G., Kumar, K., and Zhao, B. (2017). Briefing: High-performance computing for city-scale modelling and simulations. Proceedings of the Institution of Civil Engineers - Smart Infrastructure and Construction, 170(4), 80-85.

5. Chen, L. (2012). Agent-based modeling in urban and architectural research: A brief literature review. Frontiers of Architectural Research, 1(2), 166-177.

6. Zheng, H., Son, Y.-J., Chiu, Y.-C., Head, L., Feng, Y., Xi, H., Kim, S., and Hickman, M. (2013). A primer for agent-based simulation and modeling in transportation applications. United States. Federal Highway Administration.

7. Reynolds, C. W. (1987). Flocks, Herds, and Schools: A Distributed Behavioral Model. 21.

8. Abar, S., Theodoropoulos, G. K., Lemarinier, P., and O'Hare, G. M. P. (2017). Agent Based Modelling and Simulation tools: A review of the state-of-art software. Computer Science Review, 24, 13-33.

9. Boeing, G. (2017). OSMnx: New methods for acquiring, constructing, analyzing, and visualizing complex street networks. Computers, Environment and Urban Systems, 65, 126-139.

10. Zhao, B., Soga, K., and Silva, E. (2016). Simulating the degradation and maintenance effects on an integrated urban transport infrastructure system. Transforming the Future of Infrastructure through Smarter Information - Proceedings of the International Conference on Smart Infrastructure and Construction, ICSIC 2016, 609-614.

11. Li, S., Dragicevic, S., Castro, F. A., Sester, M., Winter, S., Coltekin, A., Pettit, C., Jiang, B., Haworth, J., Stein, A., and Cheng, T. (2016). Geospatial big data handling theory and methods: A review and research challenges. ISPRS Journal of Photogrammetry and Remote Sensing, Theme issue State-of-the-art in photogrammetry, remote sensing and spatial information science, 115, 119-133.

12. Ben-Akiva, M., and Bierlaire, M. (1999). Discrete Choice Methods and their Applications to Short Term Travel Decisions. Handbook of Transportation Science, R. W. Hall, ed., Springer US, Boston, MA, 5-33.
13. Çolak, S., Lima, A., and González, M. C. (2016). Understanding congested travel in urban areas. Nature Communications, 7, 10793.

14. Feygin, S., and Pozdnoukhov, A. (2018). Peer pressure enables actuation of mobility lifestyles. Transportation Research Part C: Emerging Technologies, 87, 26-45.

15. Comfort, L. K., and Haase, T. W. (2006). Communication, Coherence, and Collective Action: The Impact of. Hurricane Katrina on Communications Infrastructure. Public Works Management \& Policy, 1-16.

16. Boeing, G. (2018). A multi-scale analysis of 27,000 urban street networks: Every US city, town, urbanized area, and Zillow neighborhood. Environment and Planning B: Urban Analytics and City Science, 239980831878459.

17. Liang, W., Lam, N. S.-N., Qin, X., and Ju, W. (2015). A Two-level Agent-Based Model for Hurricane Evacuation in New Orleans. Journal of Homeland Security and Emergency Management, 12(2).

18. Wang, R.-Q., Stacey, M. T., Herdman, L. M. M., Barnard, P. L., and Erikson, L. (2018). The Influence of Sea Level Rise on the Regional Interdependence of Coastal Infrastructure. Earth's Future, 6(5), 677-688. 